\title{
$m$-noncrossing partitions and $m$-clusters
}

\author{
Aslak Bakke Buan, 杵Idun Reiten 非 and Hugh Thomas” \\ 1 Institutt for matematiske fag \\ Norges teknisk-naturvitenskapelige universitet \\ N-7491 Trondheim \\ Norway \\ ${ }^{2}$ Department of Mathematics and Statistics \\ University of New Brunswick \\ Fredericton $N B$ \\ E3B $1 J_{4}$ Canada
}

\begin{abstract}
Let $W$ be a finite crystallographic reflection group, with root system $\Phi$. Associated to $W$ there is a positive integer, the generalized Catalan number, which counts the clusters in the associated cluster algebra, the noncrossing partitions for $W$, and several other interesting sets. Bijections have been found between the clusters and the noncrossing partitions by Reading and Athanasiadis et al.

There is a further generalization of the generalized Catalan number, sometimes called the Fuss-Catalan number for $W$, which we will denote $C_{m}(W)$. Here $m$ is a positive integer, and $C_{1}(W)$ is the usual generalized Catalan number. $C_{m}(W)$ counts the $m$-noncrossing partitions for $W$ and the $m$-clusters for $\Phi$. In this abstract, we will give an explicit description of a bijection between these two sets.

The proof depends on a representation-theoretic reinterpretation of the problem, in terms of exceptional sequences of representations of quivers.

Résumé. Soit $W$ un groupe de réflections fini et crystallographique, avec système de racines $\Phi$. Associé à $W$, il y a un entier positif, le nombre de Catalan généralisé, qui compte les amas dans l'algèbre amassée associée, les partitions non-croisées de $W$, et plusieurs autres ensembles intéressantes. Des bijections entre les amas et les partitions non-croisées ont été données par Reading et Athanasiadis et al.

On peut encore généraliser le nombre de Catalan généralisé, obtenant le nombre Fuss-Catalan de $W$, que nous noterons $C_{m}(W)$. Ici $m$ est un entier positif, et $C_{1}(W)$ est le nombre Catalan généralisé standard. $C_{m}(W)$ compte les partitions $m$-non-croisées de $W$ et les $m$-amas de $\Phi$. Dans ce résumé, nous donnerons une bijection explicite entre ces deux ensembles.

La démonstration dépend d'une réinterprétation des objets du point de vue des suites exceptionnelles de représentations de carcois.
\end{abstract}

Keywords: $m$-noncrossing partitions, $m$-clusters, Fuss-Catalan numbers

\footnotetext{
†Supported by a STORFORSK-grant 167130 from the Norwegian Research Council.

¥Supported by a STORFORSK-grant 167130 from the Norwegian Research Council.

§Supported by a STORFORSK-grant 167130 from the Norwegian Research Council and an NSERC Discovery Grant.
}

1365-8050 (C) 2009 Discrete Mathematics and Theoretical Computer Science (DMTCS), Nancy, France 


\section{Fuss-Catalan numbers}

Let $W$ be a finite reflection group, with a set of simple reflections $S$ of cardinality $n$. For basic facts on reflections groups, see $\mathrm{Hu}$. We will assume throughout that $W$ is irreducible, that is to say, $W$ is not the direct product of two smaller reflection groups; all our statements generalize in a completely straightforward way to the reducible case.

A Coxeter element for $W$ is the product of the simple reflections of $W$, taken in some order. All Coxeter elements are conjugate, so they have a well-defined order, called the Coxeter number, and denoted $h$.

Associated to $W$ are a collection of positive integers called its exponents, $e_{1}, \ldots, e_{n}$. The Fuss-Catalan number for $W$ is given by the following formula:

$$
C_{m}(W)=\frac{\prod_{i=1}^{n} m h+e_{i}+1}{\prod_{i=1}^{n} e_{i}+1} .
$$

If we set $m=1$, we get the generalized Catalan number for $W$.

In the case that $W$ is the symmetric group $S_{n+1}$, the Coxeter element is an $n+1$-cycle, $h=n+1$, and the exponents are the numbers from 1 to $n$. In this case, the generalized Catalan numbers are just the usual Catalan numbers.

As we shall explain in more detail below, the Fuss-Catalan numbers count the maximal faces in the $m$-cluster complex associated to $W$ and the $m$-noncrossing partitions for $W$. Bijections have been constructed between these two sets in the $m=1$ case by Reading $[\mathrm{Re}$ and Athanasiadis et al. ABMW. Our goal in this extended abstract is to construct a bijection for arbitrary $m$.

In order for $m$-clusters and $m$-noncrossing partitions to be well-defined, we do not need to assume that $W$ is crystallographic. However, the techniques of our proof, which rely on quiver representations, do require that assumption. We will make clear at what point we have to add the crystallographic assumption.

The Fuss-Catalan numbers also arise in the study of the Shi arrangement and its generalizations (see [At]). At this point, even for $m=1$, no type-free bijection is known from either clusters or noncrossing partitions to the regions of the Shi arrangement inside the dominant chamber (which are also counted by the generalized Catalan number).

\section{Reflection group conventions}

Let $T$ be the set of all reflections for $W$. By definition, $T=\left\{w s w^{-1} \mid w \in W, s \in S\right\}$. Let $N$ be the cardinality of $T$.

Associated to $W$ is a Coxeter diagram whose vertices correspond to elements of $S$, and where two vertices are connected by an edge iff the corresponding simple reflections do not commute.

The Coxeter diagram of a finite reflection group is always a tree, so in particular it is a bipartite graph. Therefore, we can divide $S$ into two parts, $S^{+}$and $S^{-}$such that no two vertices in either part are adjacent. (This division is unique up to the labelling of the parts.) Number the reflections in $S^{+}$as $s_{1}$ to $s_{r}$, and the reflections in $S^{-}$as $s_{r+1}$ to $s_{n}$.

Fix the Coxeter element $c=s_{1} \ldots s_{n}$.

For $1 \leq i \leq N$, let $r_{i}$ be defined as $s_{1} s_{2} \ldots s_{i-1} s_{i} s_{i-1} \ldots s_{1}$, where the indexing of simple reflections is taken $\bmod n$, so that $s_{n+1}=s_{1}$, etc. 
Each reflection in $T$ occurs as $r_{i}$ for exactly one value of $i$ with $1 \leq i \leq N$. Define a total order on $T$ by saying that $r_{i}<r_{j}$ iff $i<j$.

\section{3 m-noncrossing partitions}

In this section we discuss $m$-noncrossing partitions for a reflection group $W$. The definition is due to Armstrong; for further information, see [Ar1].

Define $\ell_{T}: W \rightarrow \mathbb{N}$ by letting $\ell_{T}(w)$ be the minimal length of an expression for $w$ as a product of elements of $T$. (Note that this is not the classical length function for $W$, which would consider instead only expressions for $w$ as a product of elements of $S$.) We also note, for future use, that $\ell_{T}(c)=n$.

We can partially order $W$ as follows: $u<_{T} v$ iff there is a minimal-length expression for $v$ as a product of elements of $T$ which has a minimal-length expression for $u$ as a prefix. The usual (type-free) definition of noncrossing partitions is to take $\mathrm{NC}(W)$ to be the interval from the identity element $e$ to $c$ in this order [BW1, Be]. The number of elements of $\mathrm{NC}(W)$ is the generalized Catalan number $C_{1}(W)$.

We now give an $m$-ified version. For $w \in W$, define a minimal $k$-factorization of $w$ to be a $k$-tuple $\left(u_{0}, \ldots, u_{k-1}\right)$ of elements of $W$ such that

$$
w=u_{0} \ldots u_{k-1} \quad \text { and } \quad \ell_{T}(w)=\sum_{i} \ell_{T}\left(u_{i}\right)
$$

We define $\mathrm{NC}^{(m)}(W)$, the $m$-noncrossing partitions of $W$ to be the collection of minimal $m+1$ factorizations of $c$. (Note that there is a bijection from $\mathrm{NC}^{(1)}$ to $\mathrm{NC}$, defined by sending $(u, v)$ to $u$.)

Armstrong obtained the following enumeration of the $m$-noncrossing partitions.

Theorem 1 ([Ar1] $)\left|\mathrm{NC}^{(m)}(W)\right|=C_{m}(W)$.

\section{Coloured factorizations}

A coloured factorization of the Coxeter element $c$ is simply an expression for $c$ as a product of $n$ elements of $T$, where each reflection has an associated colour in $\mathbb{Z}$. We will write the colour as a superscript in parentheses.

We define an $m$-increasing coloured factorization to be a coloured factorization whose colours are chosen from 0 to $m$, such that the colours appear in weakly increasing order, and among the reflections of a given colour, the order of the reflections is increasing with respect to the total order on $T$.

Proposition 1 There is a bijection between m-noncrossing partitions and $m$-increasing coloured factorizations.

To construct the bijection, we use the following result:

Theorem 2 ([ABW] $)$ Let $u \leq_{T} c$, with $\ell_{T}(u)=r$. There is a unique factorization of $u$ as a product of $r$ reflections $u=t_{1} \ldots t_{r}$ such that $t_{1}<\ldots<t_{r}$. 
This factorization appears in $[\mathrm{ABW}]$ as the set of labels on the increasing chain from $e$ to $u$ in an EL-labelling for NC.

We now return to the problem of constructing an $m$-increasing factorization of $c$ from an $m$ noncrossing partition. Let $u=\left(u_{0}, \ldots, u_{m}\right)$ be an $m$-noncrossing partition. Note that $u_{i}<_{T} c$ for all $i$, so Theorem 2 applies to each $u_{i}$. Let $\left(t_{i 1}, \ldots, t_{i r_{i}}\right)$ be the factorization of $u_{i}$ obtained from Theorem 2. The $m$-increasing coloured factorization associated to $u$ is

$$
\left(t_{01}^{(0)}, \ldots, t_{0 r_{0}}^{(0)}, t_{11}^{(1)}, \ldots, t_{1 r_{1}}^{(1)}, \ldots, t_{m r_{m}}^{(m)}\right) .
$$

In other words, we take the factorizations of each of the $u_{i}$ from Theorem 2 concatenate them, and colour the reflections corresponding to $u_{i}$ with the colour $i$.

It is clear that this map from $m$-noncrossing partitions to $m$-increasing factorizations can be inverted, and thus defines a bijection.

\section{5 m-clusters}

Let $\Phi$ be a root system for $W$, with simple roots $\Pi=\left\{\alpha_{1}, \ldots, \alpha_{n}\right\}$ corresponding (in order) to the simple reflections $s_{1}, \ldots, s_{n}$. We do not (yet) assume that $\Phi$ is crystallographic. The $m$-coloured almost positive roots consist of $m$ copies of $\Phi_{>0}$, each indexed by a number from 0 to $m-1$, together with a single copy of $-\Pi$, the negative simple roots. The set of $m$-coloured almost positive roots is denoted $\Phi_{\geq-1}^{(m)}$.

The $m$-cluster complex was defined by Fomin and Reading [FR as a certain simplicial complex on this set. We will give an equivalent definition, which is due to Tzanaki $\mathrm{Tz}$ (up to some changes of convention).

Define an $m$-decreasing coloured factorization of $c$ as follows:

- The colours of the reflections are integers from 0 to $\mathrm{m}$.

- The colours appear in weakly decreasing order.

- Among the reflections of a fixed colour, the reflections appear in decreasing order with respect to the total order on $T$.

- The only reflections of colour $m$ which are allowed are $\left\{r_{i}=s_{1} s_{2} \ldots s_{i} \ldots s_{2} s_{1} \mid 1 \leq i \leq n\right\}$.

There is a bijection $\phi$ from the set of roots $\Phi_{\geq-1}^{(m)}$, to the set of coloured reflections that can appear in an $m$-decreasing factorization of $c$. It is defined as follows:

- $\phi$ sends the coloured positive $\operatorname{root} \beta^{(i)}$ to the coloured reflection $t_{\beta}^{(i)}$, where $t_{\beta}$ is the reflection through the hyperplane perpendicular to $\beta$,

- $\phi$ sends the negative simple root $-\alpha_{i}$ to the reflection $r_{i}^{(m)}$.

Note that for $s_{i} \in S^{-}, \phi\left(-\alpha_{i}\right)$ is not the reflection $s_{i}$.

The result of Tzanaki (which generalizes a result of [BW2] in the $m=1$ case), and which we can take as the definition of $m$-clusters, is the following: 


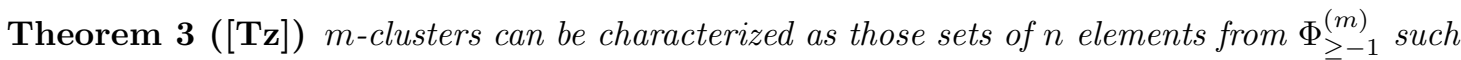
that, if their corresponding reflections under $\phi$ are ordered in decreasing order (by colour and then with respect to the total order on $T$ ), the result is an $m$-decreasing factorization of $c$.

The enumeration of $m$-clusters was carried out by Fomin and Reading:

Theorem 4 ([FR]) The number of $m$-clusters for $\Phi$ is $\mathrm{NC}^{(m)}(W)$.

We have now defined the objects which we are interested in, the $m$-noncrossing partitions for $W$ and the $m$-clusters for $\Phi$, and have recalled that they have the same cardinality. We will now proceed to define a bijection between them, or rather, between the $m$-increasing and $m$-decreasing factorizations of $c$.

\section{Mutation of coloured factorizations}

There is a mutation procedure which allows one to replace one coloured factorization of $c$ by another. The term mutation does not come from cluster algebras, but rather from the theory of exceptional sequences. See the final section for more details and references.

For $1 \leq i \leq n-1$, define an operation $\mu_{i}$ on coloured factorizations as follows.

$$
\mu_{i}\left(t_{1}^{\left(c_{1}\right)}, \ldots, t_{i}^{\left(c_{i}\right)}, t_{i+1}^{\left(c_{i+1}\right)}, \ldots, t_{n}^{\left(c_{n}\right)}\right)=\left(t_{1}^{\left(c_{1}\right)}, \ldots, t_{i+1}^{\left(c_{i+1}\right)},\left(t_{i+1} t_{i} t_{i+1}\right)^{(d)}, \ldots, t_{n}^{\left(c_{n}\right)}\right)
$$

where $d=c_{i}+1$ if $t_{i+1} t_{i} t_{i+1}<t_{i}$, otherwise $d=c_{i}$.

Lemma 1 The operations $\mu_{i}$ satisfy the braid relations, that is to say, $\mu_{i} \mu_{i+1} \mu_{i}=\mu_{i+1} \mu_{i} \mu_{i+1}$, and $\mu_{i} \mu_{j}=\mu_{j} \mu_{i}$ if $|i-j| \geq 2$.

Define $\mu_{\text {rev }}=\mu_{1}\left(\mu_{2} \mu_{1}\right)\left(\mu_{3} \mu_{2} \mu_{1}\right) \ldots\left(\mu_{n-1} \mu_{n-2} \ldots \mu_{1}\right)$. (Note that, since the $\mu_{i}$ satisfy the braid relations, there are many equivalent ways to define $\mu_{\mathrm{rev}} \cdot$ )

Then we have the following theorem:

Theorem $5 \mu_{\mathrm{rev}}$ defines a bijection from the $m$-decreasing coloured factorizations of $c$ to the $m$-increasing coloured factorizatons of $c$.

Together with the bijections we have already established between $m$-clusters and $m$-decreasing factorizations of $c$, and between $m$-noncrossing partitions and $m$-increasing factorizations of $c$, this defines a bijection between $m$-clusters and $m$-noncrossing partitions, as desired.

\section{Example: $A_{2}, m=2$}

In this section, we consider a small example. $W$ is the symmetric group on 3 letters, generated by $s_{1}=(12)$ and $s_{2}=(23)$. Let $m=2$.

$S^{+}=\left\{s_{1}\right\}, S^{-}=\left\{s_{2}\right\} . \quad c=s_{1} s_{2}=(123) . h=3$. Write $t$ for $s_{1} s_{2} s_{1}=(13)$, the unique non-simple reflection. The total order on the reflections is $s_{1}<t<s_{2}$. Write $\alpha_{1}$ and $\alpha_{2}$ for the simple roots, and $\beta$ for the unique non-simple positive root. The Fuss-Catalan number is 12.

In the table below, we list the twelve 2-clusters for $A_{2}$, their corresponding decreasing coloured factorizations as in Theorem 3, the result of applying $\mu_{\mathrm{rev}}=\mu_{1}$ to the 2-decreasing coloured factorization (which yields a 2-increasing factorization), and the corresponding 2-noncrossing partition. 


$$
\begin{aligned}
& \left\{\beta^{(0)}, \alpha_{1}^{(0)}\right\} \rightarrow\left(t^{(0)}, s_{1}^{(0)}\right) \rightarrow\left(s_{1}^{(0)}, s_{2}^{(0)}\right) \rightarrow\left(s_{1} s_{2}, e, e\right) \\
& \left\{\beta^{(1)}, \alpha_{1}^{(0)}\right\} \rightarrow\left(t^{(1)}, s_{1}^{(0)}\right) \rightarrow\left(s_{1}^{(0)}, s_{2}^{(1)}\right) \rightarrow\left(s_{1}, s_{2}, e\right) \\
& \left\{-\alpha_{2}, \alpha_{1}^{(0)}\right\} \rightarrow\left(t^{(2)}, s_{1}^{(0)}\right) \rightarrow\left(s_{1}^{(0)}, s_{2}^{(2)}\right) \rightarrow\left(s_{1}, e, s_{2}\right) \\
& \left\{\alpha_{2}^{(0)}, \beta^{(0)}\right\} \rightarrow\left(s_{2}^{(0)}, t^{(0)}\right) \rightarrow\left(t^{(0)}, s_{1}^{(1)}\right) \rightarrow\left(t, s_{1}, e\right) \\
& \left\{\alpha_{2}^{(1)}, \beta^{(0)}\right\} \rightarrow\left(s_{2}^{(1)}, t^{(0)}\right) \rightarrow\left(t^{(0)}, s_{1}^{(2)}\right) \rightarrow\left(t, e, s_{1}\right) \\
& \left\{\alpha_{1}^{(1)}, \alpha_{2}^{(0)}\right\} \rightarrow\left(s_{1}^{(1)}, s_{2}^{(0)}\right) \rightarrow\left(s_{2}^{(0)}, t^{(1)}\right) \rightarrow\left(s_{2}, t, e\right) \\
& \left\{-\alpha_{1}, \alpha_{2}^{(0)}\right\} \rightarrow\left(s_{1}^{(2)}, s_{2}^{(0)}\right) \rightarrow\left(s_{2}^{(0)}, t^{(2)}\right) \rightarrow\left(s_{2}, e, t\right) \\
& \left\{\beta^{(1)}, \alpha^{(1)}\right\} \rightarrow\left(t^{(1)}, s_{1}^{(1)}\right) \rightarrow\left(s_{1}^{(1)}, s_{2}^{(1)}\right) \rightarrow\left(e, s_{1} s_{2}, e\right) \\
& \left\{-\alpha_{2}, \alpha_{1}^{(1)} \rightarrow \rightarrow\left(t^{(2)}, s_{1}^{(1)}\right) \rightarrow\left(s_{1}^{(1)}, s_{2}^{(2)}\right) \rightarrow\left(e, s_{1}, s_{2}\right)\right. \\
& \left\{\alpha_{2}^{(1)}, \beta^{(1)}\right\} \rightarrow\left(s_{2}^{(1)}, t^{(1)}\right) \rightarrow\left(t^{(1)}, s_{1}^{(2)}\right) \rightarrow\left(e, t, s_{1}\right) \\
& \left\{-\alpha_{1}, \alpha_{2}^{(1)} \rightarrow \rightarrow\left(s_{1}^{(2)}, s_{2}^{(1)}\right) \rightarrow\left(s_{2}^{(1)}, t^{(2)}\right) \rightarrow\left(e, s_{2}, t\right)\right. \\
& \left\{-\alpha_{2},-\alpha_{1}\right\} \rightarrow\left(t^{(2)}, s_{1}^{(2)}\right) \rightarrow\left(s_{1}^{(2)}, s_{2}^{(2)}\right) \rightarrow\left(e, e, s_{1} s_{2}\right)
\end{aligned}
$$

\section{Positive parts}

There is a subcomplex of the $m$-cluster complex which is called its positive part, that is, the part which does not involve any of the negative simple roots. Under the correspondence of Theorem 3 the positive $m$-clusters (the $m$-clusters in the positive part) correspond to $m$-decreasing factorizations of $c$ in which no reflections with the colour $m$ appear.

Theorem 6 ([FR]) The number of m-clusters in the positive part of the cluster complex for $\Phi$ is:

$$
\left|C_{-m-1}(W)\right|=\frac{\prod_{i=1}^{n} m h+e_{i}-1}{\prod_{i=1}^{n} e_{i}+1} .
$$

We can give the following description of the image of the positive $m$-clusters under our bijection. We use the definition of $r_{i}$ from Section 2

Theorem 7 The image under $\mu_{\mathrm{rev}}$ of the $m$-decreasing factorizations of c corresponding to positive $m$-clusters, consists of those $m$-increasing factorizations in which the coloured reflections $\left\{r_{N-i+1}^{(m)} \mid 1 \leq i \leq n\right\}$ do not appear.

In fact, as was conjectured by Armstrong [Ar2], there is a whole family of natural bijections. In Section 2 we defined $r_{1}, \ldots, r_{N}$. We will now extend that definition. For $i \geq 1$, define $r_{i}$ to be the coloured reflection $\left(s_{1} s_{2} \ldots s_{i} \ldots s_{1}\right)^{(\lfloor i / N\rfloor)}$.

Totally order the coloured reflections by $r_{i}<r_{j}$ iff $i<j$. Define a decreasing coloured factorization of $c$ to be a factorization of $c$ into coloured reflections such that the factors are decreasing with respect to this order, and define an increasing coloured factorization of $c$ similarly.

Then Proposition 1 can be restated as saying that $m$-noncrossing partitions are in bijection with increasing factorizations of $c$ using coloured reflections from the set $\left\{r_{1}, \ldots, r_{(m+1) N}\right\}$. Theorem 3 can be restated as saying that $m$-clusters are in bijection with decreasing factorizations of $c$ using coloured reflections from the set $\left\{r_{1}, \ldots, r_{m N+n}\right\}$, while the positive $m$-clusters are in bijection with decreasing factorizations using coloured reflections from the set $\left\{r_{1}, \ldots, r_{m N}\right\}$.

We have the following generalization of Theorems 5 and 7 . 
Theorem 8 For any $0 \leq m$ and $0 \leq k$, the image under $\mu_{\mathrm{rev}}$ of the decreasing factorizations of $c$ using coloured reflections $\left\{r_{i}\right\}$ with $1 \leq i \leq N m+(k+1) n$, consists of the increasing factorizations of c using coloured reflections $\left\{r_{i}\right\}$ with $1 \leq i \leq N(m+1)+k n$.

Other than the cases described by Theorems 5 and 7 , there do not seem to be enumerative results known for these families.

\section{Representation theory}

In this section, we shall sketch the approach taken in our proofs of the preceding results. This approach depends heavily on the theory of quiver representations, of which we will attempt to sketch some elements. The interested reader is urged to consult [ARS, ASS] for an accessible introduction to this topic.

Assume that $W$ is a finite, simply laced reflection group, with root system $\Phi$. (We shall discuss more general settings at the end of the section.) Let $Q$ be the directed graph obtained by taking the Coxeter diagram of $W$ and orienting the edges from $S_{-}$to $S_{+}$. Fix an algebraically closed ground field $k$.

A representation $V$ of $Q$ is an assignment of a finite dimensional vector space $V_{i}$ over $k$ to each vertex $i$ of $Q$, and a linear map $V_{\alpha}$ between the corresponding vector spaces to each arrow $\alpha$ of $Q$. A morphism from $V$ to $W$ is a collection of linear maps $f_{i}: V_{i} \rightarrow W_{i}$ which makes all squares commute. The representations of $Q$ form an abelian category, which is denoted $\operatorname{rep}(Q)$. This category is equivalent to the category of finitely generated modules over the path algebra of $Q$.

If $V, W \in \operatorname{rep}(Q)$, we can define a $k$-vector space $\operatorname{Hom}(V, W)$. Using standard homological algebra, one can then $\operatorname{define}_{\operatorname{Ext}^{i}}(V, W)$ for $i>0$. Note that $\operatorname{rep}(Q)$ is hereditary, that is to say, $\operatorname{Ext}^{i}(V, W)=0$ for $i \geq 2$.

A representation of $Q$ is called indecomposable if it is not the direct sum of two subrepresentations. By Gabriel's theorem, the indecomposable representations of $Q$ are naturally in 1-1 correspondence with $\Phi_{>0}$, or, equivalently, with $T$. (If $Q$ is non-Dynkin, the situation is more complex.)

Following $\mathrm{Cr}$, define an exceptional sequence of representations of $Q$ to be a sequence of indecomposable representations $\left(F_{1}, \ldots, F_{r}\right)$ such that $\operatorname{Hom}\left(F_{i}, F_{j}\right)=0=\operatorname{Ext}^{1}\left(F_{i}, F_{j}\right)$ for $i<j$. (Note that this reverses the usual convention for the order of an exceptional sequence. Also, one normally must also require that $\operatorname{Ext}^{1}\left(F_{i}, F_{i}\right)=0$ for all $i$, but this is automatic in the present setting where $Q$ is Dynkin.) The maximal length of an exceptional sequence is $n$.

The notion of exceptional sequence is related to the concepts we have been discussing via the following theorem:

Theorem $9([\mathbf{I T}])$ For $\beta_{1}, \ldots, \beta_{n}$ a collection of $n$ positive roots, $\left(E_{\beta_{1}}, \ldots, E_{\beta_{n}}\right)$ is an exceptional sequence iff $t_{\beta_{1}} \ldots t_{\beta_{n}}=c$.

(This theorem is shown in [T] in the case which we need here, when $Q$ is Dynkin, and also when $Q$ is affine; for arbitrary $Q$ without oriented cycles, it is proved in [IS.)

There are well-defined mutation operations on the set of exceptional sequences of a given length. Given an exceptional sequence $\left(E_{1}, \ldots, E_{n}\right)$, which, for convenience, we assume to have maximal length, for $1 \leq i \leq n-1$, the operation $\mu_{i}$ is defined by: 


$$
\mu_{i}\left(E_{1}, \ldots, E_{i}, E_{i+1}, \ldots, E_{n}\right)=\left(E_{1}, \ldots, E_{i+1}, M, \ldots, E_{n}\right)
$$

where $M$ is uniquely determined by the fact that $\mu_{i}\left(E_{1}, \ldots, E_{n}\right)$ forms an exceptional sequence.

Now, consider the collection of all factorizations of $c$ as a product of $n$ reflections. Clearly, there is also a mutation operation on such factorizations: just consider the mutation operation from Section 6, but ignore colour. It is a theorem of $\mathrm{Cr}$ that mutation of exceptional sequences can also be defined in more Coxeter-theoretic terms, from which it follows that mutation of exceptional sequences agrees via Theorem 9 with the mutation operation which we have just defined on factorizations of $c$.

In order to interpret coloured factorizations representation-theoretically, we must pass from $\operatorname{rep}(Q)$ to its bounded derived category $D^{b}(Q)$. As usual, we think of $\operatorname{rep}(Q)$ sitting inside $D^{b}(Q)$ in degree 0 . Thanks to the shift functor [1] of $D^{b}(Q)$, for any indecomposable $V \in \operatorname{rep}(Q)$, we have indecomposable objects $V[i] \in D^{b}(Q)$ for all $i \in \mathbb{Z}$. Because $\operatorname{rep}(Q)$ is hereditary, there are no other indecomposable objects in $D^{b}(Q)$. Thus, there is a bijection between coloured reflections and indecomposable objects in $D^{b}(Q)$. The notions of exceptional sequences and mutations extend naturally to $D^{b}(Q)$, and these mutation operations agree precisely with those of Section 6.

Next, one has to study the special types of exceptional sequences which correspond to $m$ increasing and $m$-decreasing coloured factorizations. One has:

Proposition 2 If $\left(t_{1}^{\left(c_{1}\right)}, \ldots, t_{n}^{\left(c_{n}\right)}\right)$ is a coloured factorization of $c$, and $\left(E_{1}, \ldots, E_{n}\right)$ is the corresponding exceptional sequence in $D^{b}(Q)$, then the factorization is $m$-increasing iff:

- For all $i, E_{i} \in \operatorname{rep}(Q)[k]$ for some $0 \leq k \leq m$,

- For all $i \neq j, \operatorname{Ext}^{k}\left(E_{i}, E_{j}\right)=0$ for $-m \leq k \leq 0$.

Proposition 3 If $\left(t_{1}^{\left(c_{1}\right)}, \ldots, t_{n}^{\left(c_{n}\right)}\right)$ is a coloured factorization of $c$, and $\left(E_{1}, \ldots, E_{n}\right)$ is the corresponding exceptional sequence in $D^{b}(Q)$, then the factorization is $m$-decreasing iff:

- For all $i, E_{i} \in \operatorname{rep}(Q)[k]$ for some $0 \leq k<m$ or $E_{i}=P[m]$ for some indecomposable projective $P$,

- For all $i, j, \operatorname{Ext}^{k}\left(E_{i}, E_{j}\right)=0$ for $1 \leq k \leq m$.

Note that this latter proposition is closely related to the usual approach to categorifying the $m$-cluster combinatorics of [FR], see [Zh, $\mathrm{Th}, \mathrm{Wr}$ and subsequent papers.

Theorem 5 is then proved by showing that $\mu_{\text {rev }}$ transforms the exceptional sequences of Proposition 3 into those of Proposition 2 Theorems 7 and 8 are proved similarly.

If $W$ is a non-simply laced but crystallographic reflection group, then our techniques can be made to apply by a folding argument, or by working over a non-algebraically closed ground field and applying [Ri]. If $W$ is non-crystallographic, our techniques do not apply. Note that the definition we have given of the bijection from $m$-increasing factorizations of $c$ to $m$-decreasing factorizations of $c$ still makes sense, but we cannot prove that it is a bijection.

There is nothing in our approach which really requires that $W$ be finite; all we really need is the much weaker condition that $Q$ have no oriented cycles. In this much more general setting, 
however, there are some additional aspects which must be taken care of. We are preparing a paper in which we will explain these extra aspects, and provide the proofs of the assertions in this extended abstract.

\section{Acknowledgements}

The third author would like to thank Drew Armstrong and Colin Ingalls for helpful conversations. Much of this work was carried out while the third author was visiting the first two authors at the Institutt for Matematiske Fag at NTNU; he thanks them for their hospitality. The authors would also like to thank the anonymous referees for their helpful comments.

\section{References}

[Ar1] D. Armstrong, Generalized noncrossing partitions and combinatorics of Coxeter groups. Mem. AMS, to appear. arXiv:0712.1047.

[Ar2] D. Armstrong, personal communication, 2006.

[ASS] I. Assem, D. Simson, and A. Skowroński, Elements of the representation theory of associative algebras, vol. 1. Cambridge University Press, Cambridge, 2006.

[At] C. Athanasiadis, Generalized Catalan numbers, Weyl groups and arrangements of hyperplanes. Bull. London Math. Soc. 36 (2004), 294-302.

[ABW] C. Athanasiadis, T. Brady, and C. Watt, Shellability of noncrossing partition lattices. Proc. Amer. Math. Soc. 135 (2007), no. 4, 939-949.

[ABMW] C. Athanasiadis, T. Brady, J. McCammond, and C. Watt, $h$-vectors of generalized associahedra and noncrossing partitions. Int. Math. Res. Not. 2006, Art. ID 69705, 28 pp.

[ARS] M. Auslander, I. Reiten, and S. Smalø, Representation theory of Artin algebras. Cambridge Studies in Advanced Mathematics 36. Cambridge University Press, Cambridge, 1997.

[Be] D. Bessis, The dual braid monoid. Ann. Sci. École Norm. Sup. (4) 36 (2003), 647-683.

[BW1] T. Brady and C. Watt, $K(\pi, 1)$ s for Artin groups of finite type. Geom. Dedicata 94 (2002) 225-250.

[BW2] T. Brady and C. Watt, Lattices in finite real reflection groups, Trans. Amer. Math. Soc. 360 (2008), no. 4, 1983-2005.

[Cr] W. Crawley-Boevey, Exceptional sequences of representations of quivers. Proceedings of the Sixth International Conference on Representations of Algebras (Ottawa, ON, 1992), 7 pp., Carleton-Ottawa Math. Lecture Note Ser. 14, Carleton Univ., Ottawa, ON, 1992. 
[FR] S. Fomin and N. Reading, Generalized cluster complexes and Coxeter combinatorics. Int. Math. Res. Not. 2005, no. 44, 2709-2757.

$[\mathrm{Hu}] \quad$ J. Humphreys, Reflection groups and Coxeter groups. Cambridge University Press, Cambridge, 1990.

[IS] K. Igusa and R. Schiffler, Exceptional sequences and clusters, with an appendix by the authors and H. Thomas, arXiv:0901.2590.

[IT] C. Ingalls and H. Thomas, Noncrossing partitions and representations of quivers. Compositio Math., to appear. arXiv:math/0612219.

[Re] N. Reading, Coxeter-sortable elements and noncrossing partitions. Trans. Amer. Math. Soc. 359 (2007), no. 12, 5931-5958.

[Ri] C. Ringel, The braid group action on the set of exceptional sequences of a hereditary Artin algebra. Abelian group theory and related topics (Oberwolfach, 1993), 339-352, Contemp. Math. 171, Amer. Math. Soc., Providence, RI, 1994.

[Th] H. Thomas, Defining an m-cluster category. J. Algebra 318 (2007), no. 1, 37-46.

$[\mathrm{Tz}] \quad$ E. Tzanaki, Faces of generalized cluster complexes and noncrossing partitions. SIAM J. Discrete Math. 22 (2008), no. 1, 15-30.

[Wr] A. Wraalsen, Rigid objects in higher cluster categories. J. Algebra, to appear. arXiv:math/0712.2970.

[Zh] B. Zhu, Generalized cluster complexes via quiver representations. J. Algebraic Combin. 27 (2008), no. 1, 35-54. 